\title{
On affine functions with respect to some means
}

\author{
AgATa NowAK
}

Abstract. The purpose of the present paper is to investigate the functional equation

$$
M(f(x), g(y))=h(N(x, y)),
$$

where $f, g$ and $h$ are self-mappings of a real interval $I$ and $M, N: I^{2} \rightarrow I$ are functions. In particular, we will show that under appropriate assumptions imposed on the functions $M, N$ the local boundedness of $f$ implies the continuity of $g$.

Mathematics Subject Classification (2000). 26E60 (26B25 39B52).

Keywords. Mean, logarithmic mean, M-affine function, M-convex function, functional equation.

\section{Introduction: basic definitions and auxiliary lemmas}

In this paper $I$ will always denote a non-degenerate interval contained in $\mathbb{R}$.

A function $M: I^{2} \rightarrow I$ is called a mean on $I$ if

$$
\min \{x, y\} \leq M(x, y) \leq \max \{x, y\}, \quad x, y \in I .
$$

A mean $M$ is called a strict mean if

$$
\min \{x, y\}<M(x, y)<\max \{x, y\}, \quad x, y \in I, x \neq y .
$$

Now, let $M$ be a mean on $I$ and let $f: I \rightarrow I$. We say that $f$ is affine with respect to $M$ (or shortly $M$-affine) if

$$
f(M(x, y))=M(f(x), f(y)), \quad x, y \in I .
$$

Let us also introduce the following notation. For nonempty sets $X, Y, Z$, $u \in X, v \in Y$ and a function $F: X \times Y \rightarrow Z$, we define functions $F_{u}: Y \rightarrow Z$, $F^{v}: X \rightarrow Z$ by the formulas:

$$
\begin{array}{ll}
F_{u}(y):=F(u, y), & y \in Y \\
F^{v}(x):=F(x, v), & x \in X .
\end{array}
$$


The present paper is devoted to the following functional equation:

$$
M(f(x), g(y))=h(N(x, y)), \quad x, y \in I,
$$

where $M$ and $N$ are given functions, $M, N: I^{2} \rightarrow I$, whereas $f, g$ and $h$ are unknown functions, $f, g, h: I \rightarrow I$. Our results are analogous to the ones of $\mathrm{Ng}$ [1], who has investigated the functional equation

$$
f(x)+g(y)=h(T(x, y)),
$$

where the unknown functions $f, g$ and $h$ act on a connected or a locally connected topological space $X$, and $T: X^{2} \rightarrow X$ is a given function. The methods we apply to (1.1) are modifications of those used by $\mathrm{Ng}$ in [1].

In particular, our results cover the case of $L$-affine functions, where $L$ is a logarithmic mean, i.e.

$$
L(x, y):= \begin{cases}\frac{x-y}{\ln x-\ln y}, & x \neq y \\ x, & x=y\end{cases}
$$

which was investigated by Matkowski in [2].

Let us start with a technical lemma.

Lemma 1. Let $M: I^{2} \rightarrow I$ be a function such that for all $u, v \in I$ the mapping $M_{u}$ is strictly increasing, and the mapping $M^{v}$ is increasing and continuous. Then, for all $s, S, v_{0} \in I$ such that $s \leq S$ and $\varepsilon>0$, there exists $\delta>0$ such that for all $u, \bar{u}, v \in I$ satisfying $M(u, v)=M\left(\bar{u}, v_{0}\right), \bar{u}-u \leq \delta$ and $s \leq u \leq S$, we have $v-v_{0} \leq \varepsilon$.

Proof. Suppose (in search of a contradiction) that there exist $s, S, v_{0} \in I$ such that $s \leq S$ and

$$
\exists_{\varepsilon>0} \forall_{\delta>0} \exists_{u, \bar{u}, v \in I} \quad\left(M(u, v)=M\left(\bar{u}, v_{0}\right), \bar{u}-u \leq \delta, s \leq u \leq S, v-v_{0}>\varepsilon\right) .
$$

In particular, for every $n \in \mathbb{N}$ there exist $u_{n}, \bar{u}_{n}, v_{n} \in I$ fulfilling the conditions:

$$
M\left(u_{n}, v_{n}\right)=M\left(\bar{u}_{n}, v_{0}\right), \bar{u}_{n}-u_{n} \leq \frac{1}{n}, s \leq u_{n} \leq S, v_{n}-v_{0}>\varepsilon .
$$

Observe that the sequence $\left(u_{n}\right)_{n \in \mathbb{N}}$ has to have a convergent subsequence, thus we may assume that the sequence is convergent to some $u \in I$.

If $u_{n}+\frac{1}{n} \in I$ for infinitely many $n \in \mathbb{N}$, then we deduce the following inequalities:

$$
M\left(u_{n}, v_{0}+\varepsilon\right) \leq M\left(u_{n}, v_{n}\right)=M\left(\bar{u}_{n}, v_{0}\right) \leq M\left(u_{n}+\frac{1}{n}, v_{0}\right) .
$$

On letting $n$ tend to infinity, we get that $M\left(u, v_{0}+\varepsilon\right) \leq M\left(u, v_{0}\right)$, which contradicts the fact that $M_{u}$ is strictly increasing.

If $u_{n}+\frac{1}{n} \in I$ holds only for finitely many $n \in \mathbb{N}$, then $u=S \in I$ is the right endpoint of the interval $I$. Then we have:

$$
M\left(u_{n}, v_{0}+\varepsilon\right) \leq M\left(u_{n}, v_{n}\right)=M\left(\bar{u}_{n}, v_{0}\right) \leq M\left(u, v_{0}\right)
$$


and letting $n$ tend to infinity, we get that $M\left(u, v_{0}+\varepsilon\right) \leq M\left(u, v_{0}\right)$, which contradicts again the fact that $M_{u}$ is strictly increasing.

The proof of the next lemma is analogous and therefore we omit it here.

Lemma 2. Let $M: I^{2} \rightarrow I$ be a function such that for all $u, v \in I$ the mapping $M_{u}$ is strictly increasing, and the mapping $M^{v}$ is increasing and continuous. Then, for all $s, S, v_{0} \in I$ such that $s \leq S$ and $\varepsilon>0$, there exists $\delta>0$ such that for all $u, \bar{u}, v \in I$ satisfying $M(u, v)=M\left(\bar{u}, v_{0}\right), u-\bar{u} \leq \delta$ and $s \leq \bar{u} \leq S$, we have $v_{0}-v \leq \varepsilon$.

In the proof of our main result we also need the following lemma:

Lemma 3. [3] Let $X$ be a connected and locally connected space, $\theta: X \rightarrow \mathbb{R} a$ continuous function and $t_{1}, t_{2} \in \theta(X), t_{1}<t_{2}$. Then there exists a connected subset $B \subseteq \theta^{-1}\left(\left(t_{1}, t_{2}\right)\right)$ such that $\theta(B)=\left(t_{1}, t_{2}\right)$.

\section{Main results}

Now let us state and prove our main result:

Theorem 1. Let $M, N: I^{2} \rightarrow I$ be functions such that for all $u, v \in I$ the mapping $M_{u}$ is strictly increasing, the mapping $M^{v}$ is strictly increasing and continuous, and the mappings $N_{u}, N^{v}$ are continuous. Assume that a triple $(f, g, h): I \rightarrow I^{3}$ is a solution of (1.1) and there exists a subinterval $I_{0} \subseteq I$ such that $f$ is nonconstant on $I_{0}$ and $f\left(I_{0}\right) \subseteq[s, S]$ for some $s, S \in I$. Then $g$ is continuous.

Proof. Choose $x_{1}, x_{2} \in I_{0}$ such that $f\left(x_{1}\right) \neq f\left(x_{2}\right)$. From (1.1) and the strict monotonicity of $M^{g(y)}$ it follows that $N\left(x_{1}, y\right) \neq N\left(x_{2}, y\right)$ for all $y \in I$.

Fix $y_{0} \in I$ arbitrarily. We will prove the continuity of $g$ at $y_{0}$ using Lemmas 1 and 2 for $v_{0}=g\left(y_{0}\right)$. Let $\varepsilon>0$; there exists a $\delta>0$ such that the conditions of Lemmas 1 and 2 hold. Denote $l_{1}:=N\left(x_{1}, y_{0}\right)$ and $l_{2}:=N\left(x_{2}, y_{0}\right)$. We may assume that $l_{1}<l_{2}$. According to Lemma 3 applied to $X=I_{0}, \theta=N^{y_{0}}, t_{1}=l_{1}, t_{2}=l_{2}$ there exists an interval $B \subseteq I_{0}$ such that $N\left(B, y_{0}\right)=\left(l_{1}, l_{2}\right)$. The function $f$ is bounded on $I_{0}$ and $B \subseteq I_{0}$, so $\sup \{f(x): x \in B\}<+\infty$. Hence, $f\left(x_{0}\right) \geq f(x)-\delta$ for some $x_{0} \in B$ and for all $x \in B$.

Let $V:=\left\{y \in I: l_{1}<N\left(x_{0}, y\right)<l_{2}\right\}$. It is easy to notice that $y_{0} \in V$ and $V$ is open, since $N_{x_{0}}$ is continuous. For arbitrary $y \in V$ we have $N\left(x_{0}, y\right) \in$ $\left(l_{1}, l_{2}\right)=N\left(B, y_{0}\right)$, so there exists an $x \in B$ such that $N\left(x_{0}, y\right)=N\left(x, y_{0}\right)$. By virtue of (1.1) we get $M\left(f\left(x_{0}\right), g(y)\right)=M\left(f(x), g\left(y_{0}\right)\right)$. Apply Lemma 1 to $v_{0}:=g\left(y_{0}\right), s, S, \bar{u}:=f(x), u:=f\left(x_{0}\right), v:=g(y)$ to get that $g(y)-g\left(y_{0}\right) \leq \varepsilon$ for $y \in V$. 
Since $N\left(x_{0}, y_{0}\right) \in\left(l_{1}, l_{2}\right)=N\left(B, y_{0}\right)$ we can find points $x_{3}, x_{4} \in B$ fulfilling $N\left(x_{3}, y_{0}\right)<N\left(x_{0}, y_{0}\right)<N\left(x_{4}, y_{0}\right)$. Define the set $W$ by

$$
W:=\left\{y \in I: N\left(x_{3}, y\right)<N\left(x_{0}, y_{0}\right)<N\left(x_{4}, y\right)\right\} .
$$

One can check that $W$ is a neighborhood of $y_{0}$. Moreover, for each $y \in W$ the set $N^{y}\left(\left[\min \left\{x_{3}, x_{4}\right\}, \max \left\{x_{3}, x_{4}\right\}\right]\right)$ is an interval which contains the points $N\left(x_{3}, y\right)$ and $N\left(x_{4}, y\right)$. Therefore, the point $N\left(x_{0}, y_{0}\right)$, which lies between them, belongs to that set. Thus, for every $y \in W$ there exists a point $x$ between $x_{3}$ and $x_{4}$, which fulfills $N\left(x_{0}, y_{0}\right)=N(x, y)$. But such a point $x$ must belong to $B$, since $B$ is connected and $x_{3}, x_{4} \in B$. From (1.1) we get $M\left(f\left(x_{0}\right), g\left(y_{0}\right)\right)=$ $M(f(x), g(y))$. From Lemma 2 it follows that $g\left(y_{0}\right)-g(y)<\varepsilon$ for $y \in W$.

Thus, $g$ is continuous at $y_{0}$. Since $y_{0}$ is arbitrarily chosen, $g$ is continuous.

Remark 1. Let us note that if $I$ is compact, then the assumption of the boundedness of $f$ by elements from $I$ is obviously fulfilled. Similarly, if $f$ is continuous in some point $x_{0}$ and $f\left(x_{0}\right) \in$ int $I$, then $f$ is bounded on some neighborhood of $x_{0}$ by elements from $I$.

Proof. If $I=[a, b]$ and $f: I \rightarrow I$, then the assumptions of the previous theorem are fulfilled for $s=a, S=b, I_{0}=I$.

If $f$ is continuous in some point $x_{0}$ and $f\left(x_{0}\right) \in$ intI, then there exist $s, S \in$ intI such that $s<f\left(x_{0}\right)<S$ and a non-degenerate interval $I_{0} \subseteq I$ such that $x_{0} \in I_{0}$ and $f\left(I_{0}\right) \subseteq[s, S]$.

In the following corollary we will show that under auxiliary assumptions imposed on functions $M, N$ and if $f=g$, then it is enough to assume that $f$ is bounded from one side on some open subset of $I$ by an element from $I$.

Corollary 1. Let $M, N: I^{2} \rightarrow I$ be functions such that for all $u, v \in I$ the mapping $M_{u}$ is strictly increasing, the mapping $M^{v}$ is strictly increasing and continuous and the mappings $N_{u}, N^{v}$ are strictly increasing, continuous and $N(x, x)=x$ for every $x \in I$. If a pair $(f, h): I \rightarrow I^{2}$ is a solution of the equation

$$
h(N(x, y))=M(f(x), f(y)), \quad x, y \in I
$$

and there exist a subinterval $I_{0} \subseteq I$ and a constant $S \in I$ (or $s \in I$ ) such that $f\left(I_{0}\right) \subseteq(-\infty, S]$ (or $f\left(I_{0}\right) \subseteq[s,+\infty)$, respectively) and $f$ is non-constant on $I_{0}$ and $M^{S}$ is onto $I$, then $f$ is continuous.

Proof. We show that the function $f$ fulfilling the assumptions of the corollary also fulfills the assumptions of Theorem 1.

Let $f$ be bounded from above on an interval $I_{0}=[a, b] \subseteq I$ by a constant $S \in I$ and let $x_{0}:=\frac{1}{2}(a+b)$. We will prove that there exists a point $x_{1} \in\left(a, x_{0}\right)$ such that for every $x \in\left(x_{1}, x_{0}\right)$ there exists $y \in(a, b)$ such that $N(x, y)=x_{0}$. First, we show that there exists a pair $\left(x_{1}, y_{1}\right) \in\left(a, x_{0}\right) \times(a, b)$ 
such that $N\left(x_{1}, y_{1}\right)=x_{0}$. If there were no such pair, then we would have $N(x,(a, b)) \subseteq\left(a, x_{0}\right)$ for each $x \in\left(a, x_{0}\right)$. We infer that for every $\hat{x} \in\left(x_{0}, b\right)$

$$
x_{0}=N\left(x_{0}, x_{0}\right)<N\left(x_{0}, \hat{x}\right)=\lim _{x \rightarrow x_{0}^{-}} N(x, \hat{x}) \leq x_{0},
$$

which is impossible. Let $\left(x_{1}, y_{1}\right) \in\left(a, x_{0}\right) \times(a, b)$ be a pair for which $N\left(x_{1}, y_{1}\right)=$ $x_{0}$. For every $x \in\left(x_{1}, x_{0}\right)$ we get

$$
N\left(x, x_{0}\right)<N\left(x_{0}, x_{0}\right)=x_{0}, \quad N\left(x, y_{1}\right)>N\left(x_{1}, y_{1}\right)=x_{0} .
$$

It means that the interval $N(x,(a, b))$ contains a point greater than $x_{0}$ and a point smaller than $x_{0}$. Thus, there exists $y \in(a, b)$ such that $N(x, y)=x_{0}$.

Now let $x \in\left(x_{1}, x_{0}\right)$. We may find a $y \in(a, b)$ for which $N(x, y)=x_{0}$. Use Eq. (2.1) for the pair $(x, y)$ to get

$$
h\left(x_{0}\right)=h(N(x, y))=M(f(x), f(y)) \leq M(f(x), S) .
$$

Since $h\left(x_{0}\right) \in I$ and $M^{S}$ is a bijection from $I$ onto $I$, there exists exactly one $s \in I$ such that $M^{S}(s)=h\left(x_{0}\right)$. Observe that $s \leq f(x)$ for every $x \in\left(x_{1}, x_{0}\right)$. Thus, $f$ and $g=f$ satisfy the assumptions of Theorem 1 .

The proof when $f\left(I_{0}\right) \subseteq(s, \infty)$ follows in a similar fashion.

On imposing stronger assumptions upon $N$ we may eliminate the assumption of non-constancy of $f$ on every interval.

Theorem 2. Let $M, N: I^{2} \rightarrow I$ be mappings such that for all $u, v \in I$ the mapping $M_{u}$ is strictly increasing, the mapping $M^{v}$ is strictly increasing and continuous and the functions $N_{u}, N^{v}$ are continuous. Moreover, let $N$ be a symmetric strict mean. Assume that a triple $(f, g, h): I \rightarrow I^{3}$ is a solution of (1.1) and that there exist a subinterval $I_{0} \subset I$ and $s, S \in I$ such that $f\left(I_{0}\right) \subset[s, S]$. Then $g$ is continuous.

Proof. Due to Theorem 1 it is enough to consider the case when $f$ is constant on some non-degenerate subinterval $P \subseteq I$. We prove that in this case $g$ is constant on the whole domain.

Let $c:\left.\equiv f\right|_{P}$; we have

$$
\begin{aligned}
M(c, g(x)) & =M(f(y), g(x))=h(N(y, x))=h(N(x, y)) \\
& =M(f(x), g(y))=M(c, g(y)), \quad x, y \in P .
\end{aligned}
$$

Since the function $M_{c}$ is strictly monotone, $g$ is constant on $P$, say $\left.g\right|_{P} \equiv c_{1}$ for some $c_{1} \in I$. Thus, for all $x, y \in P$ we get

$$
h(N(x, y))=M(f(x), g(y))=M\left(c, c_{1}\right),
$$

which means that $\left.h\right|_{N(P \times P)}$ is constant and equal to $c_{2}:=M\left(c, c_{1}\right)$. On the other hand, $N$ is a mean, thus $N(P \times P)=P$. Thus, we have shown that $\left.h\right|_{P} \equiv c_{2}$. 
Now we will verify that $f$ is constant on $\operatorname{int} I$. Assume that $P$ is the maximal interval on which $f$ is constant and equals $c$ ( if $Q$ is any interval such that $P \subseteq Q$ and $\left.f\right|_{Q} \equiv c$ then $P=Q$ ); we will show that $\operatorname{int} P=\operatorname{int} I$. Let $\alpha, \beta \in \mathbb{R} \cup\{-\infty\} \cup\{+\infty\}$ be chosen in such a way that $\operatorname{int} I=(\alpha, \beta)$. Suppose that int $P \neq \operatorname{int} I$. Then the left end-point of $P$ is a real number $a>\alpha$ or the right end-point of $P$ is a real number $b<\beta$. Without loss of generality we may assume that $b<\beta$. Choose $d$ so that $[d, b) \subseteq P$. We have $\left(b, b+\frac{1}{n}\right) \subseteq I$ for sufficiently large $n \in \mathbb{N}$. For such $n$ we may choose $y_{n} \in\left(b, b+\frac{1}{n}\right)$ such that $f\left(y_{n}\right) \neq c$. The mean $N$ is strict, so $d<N(d, b)<b$. Moreover, we have $N(d, u) \in(d, b)$ for all points $u$ taken from some neighbourhood of $b$ because the function $N_{d}$ is continuous. Thus, $N\left(d, y_{k}\right) \in[d, b)$ for some $k$ in $\mathbb{N}$. On the other hand,

$$
M\left(f\left(y_{k}\right), c_{1}\right)=M\left(f\left(y_{k}\right), g(d)\right)=h\left(N\left(y_{k}, d\right)\right)=c_{2} .
$$

Thus, $f\left(y_{k}\right)=c$, because $M\left(c, c_{1}\right)=c_{2}$ and the function $M^{c_{1}}$ is strictly increasing. Thus, we get a contradiction.

Obviously, $\left.g\right|_{\operatorname{int} I} \equiv c_{1}$ and $\left.h\right|_{\operatorname{int} I} \equiv c_{2}$.

To complete the proof it remains to show that if any of the end-points of $I$ belongs to $I$, then in this edge $g$ is also equal to $c_{1}$. For example, if $\beta \in I$, then from Eq. (2.1) applied to $x \in(\alpha, \beta)$ and $y=\beta$ we get

$$
c_{2}=h(N(x, \beta))=M(f(x), g(\beta))=M(c, g(\beta)),
$$

thus, $g(\beta)=c_{1}$. If $\alpha \in I$, we proceed in a similar way.

In what follows we apply the just proved theorems to $M$-affine functions. From results of $\mathrm{Ng}$ [1] it follows that an $M$-affine function defined on $I$ and bounded from both sides has to be continuous. From the previous results it follows that two-sided boundedness of $f$ may be weakened to one-side boundedness.

Corollary 2. If $M$ is a symmetric, strict mean such that the functions $M_{u}, M^{v}$ are continuous and strictly increasing for every $u, v \in I$ and $f$ is an $M$-affine function, then the local boundedness of $f$ from one side by an element from $I$ implies its continuity.

In particular, setting the logarithmic mean for $M$ we obtain a slightly more general result than those of Matkowski [2, Lemma 2].

Corollary 3. Assume that $I$ is contained in $(0,+\infty)$ and $L$ is the logarithmic mean. If $f: I \rightarrow I$ is $L$-affine, bounded at a point from one side by an element from $I$ then $f$ is continuous.

Open Access. This article is distributed under the terms of the Creative Commons Attribution Noncommercial License which permits any noncommercial use, distribution, and reproduction in any medium, provided the original author(s) and source are credited. 


\title{
References
}

[1] Ng, C.T.: Local boundedness and continuity for a functional equation on topological spaces. Proc. Am. Math. Soc. 39, 525-529 (1973)

[2] Matkowski, J.: Affine and convex functions with respect to the logarithmic mean. Colloq. Math. 95(2), 217-230 (2003)

[3] Pfanzgl, J.: On a functional equation related to families of exponential probability measures. Aequationes Math. 4, 139-142 (1970)

\author{
Agata Nowak \\ Institute of Mathematics \\ University of Silesia \\ Bankowa 14 \\ 40-007 Katowice \\ Poland \\ e-mail: tnga@poczta.onet.pl
}

Received: August 12, 2009

Revised: May 12, 2010 